\title{
Cluster Node Computing for Target Generation Systems in Aircraft Simulations
}

\author{
Spencer Monheim, Michael Feher, James Murphy \\ NASA Ames Research Center
}

Bonnie Andro-Avila

MIRACORP, Inc. 
Discuss the development of a general purpose prototype target generator for air traffic management simulations in order to accommodate future research at NASA Ames Research Center's Aviation Systems Division 
- Definition of a Target Generator

- System Design

- System Design Philosophy

- Rationale for Language Used

- System Architecture

- Rationale for Networking Protocols Used

- Performance Results 


\section{Nast Background: What is a Target Generator?}

- Simulates multiple aircraft to create an airspace simulation

- Fly aircraft along pre-defined routes or along vectors

- Publish the positional and attitude data to an external interface

- Provides interfaces for client software such as pseudopilot or controller interfaces

- Facilitates research simulations
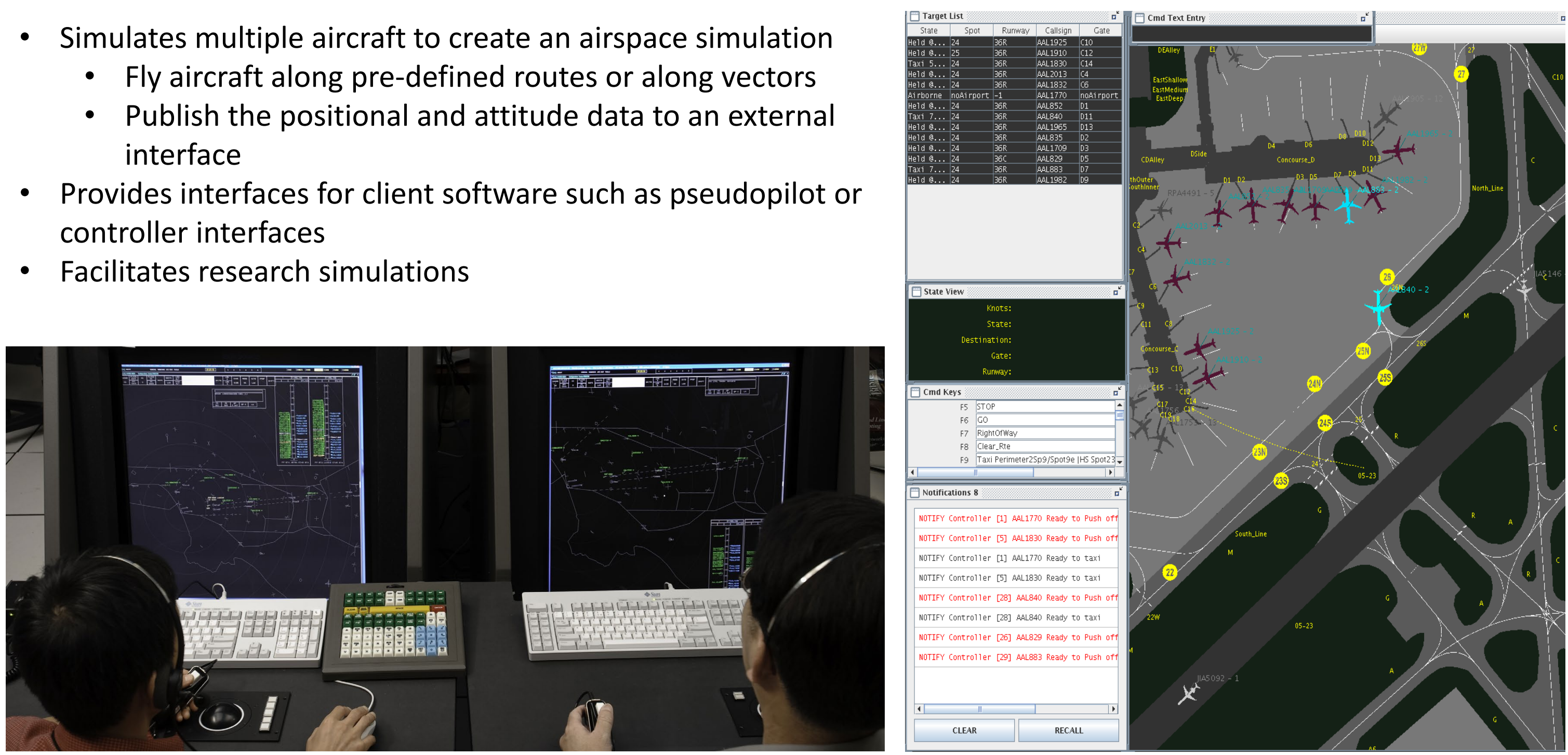
- Air traffic control simulation facility

- 360 degree visualization

- Facilitates air traffic management research simulations
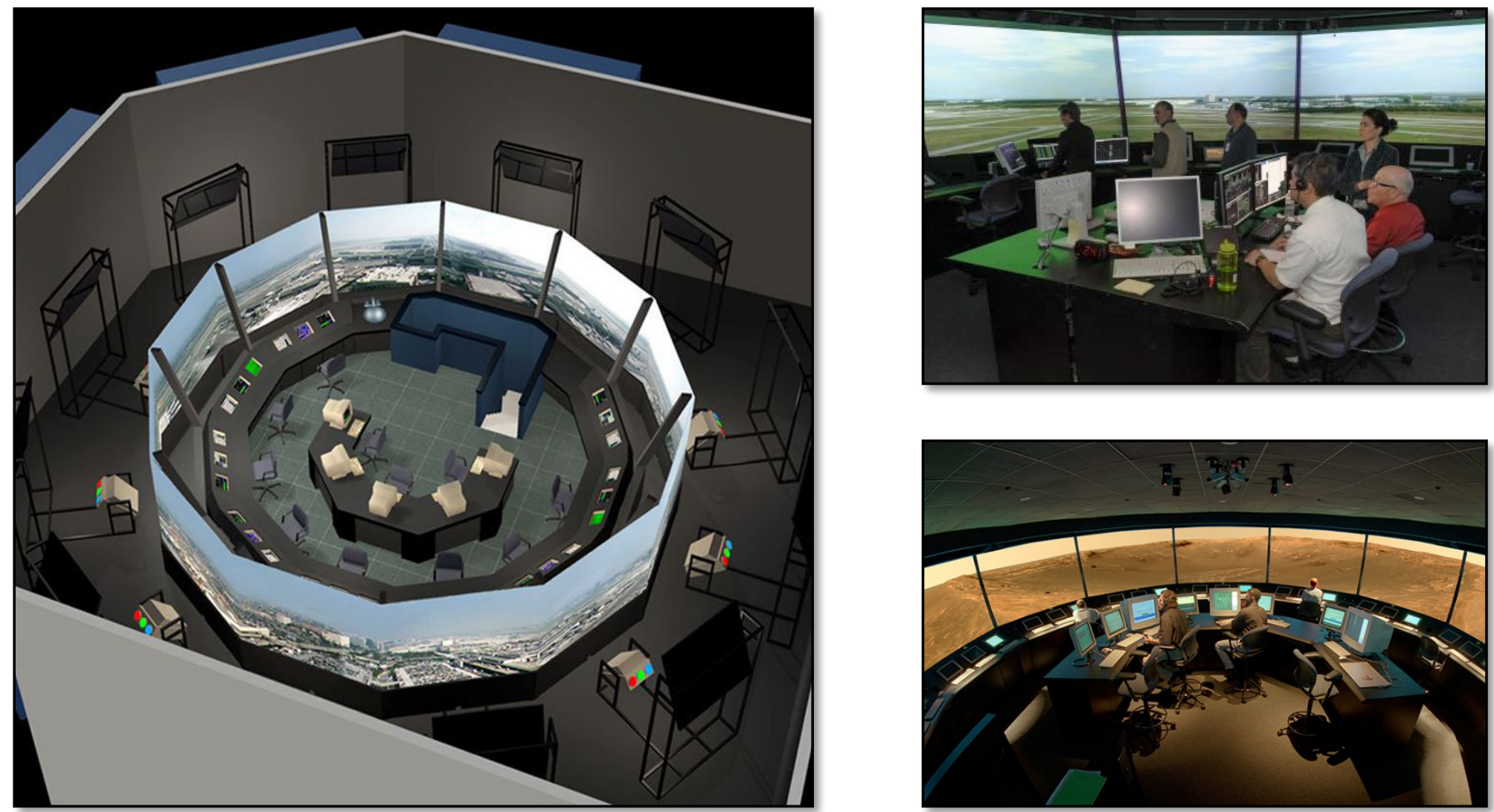
- Leverage the fact that newer processors are more commonly increasing the number of cores over single-core performance

- Separate the target generator entirely from client software (pilot stations, etc.) and utilize the UNIX-style approach to software

- Provide the capability for pilot and controller interfaces to be remotely accessible via a web browser 
NAsA Building a Target Generator Prototype - Part 2: Language

Programming Language Chosen: Go

Selection Criteria:

- Native support for multi-threading

- High developer velocity

- Support multiple network protocols like TCP, UDP, UNIX Domain Sockets, http, Websockets, etc. 


\section{NAsa Using Go's concurrency model to implement Parallelism}

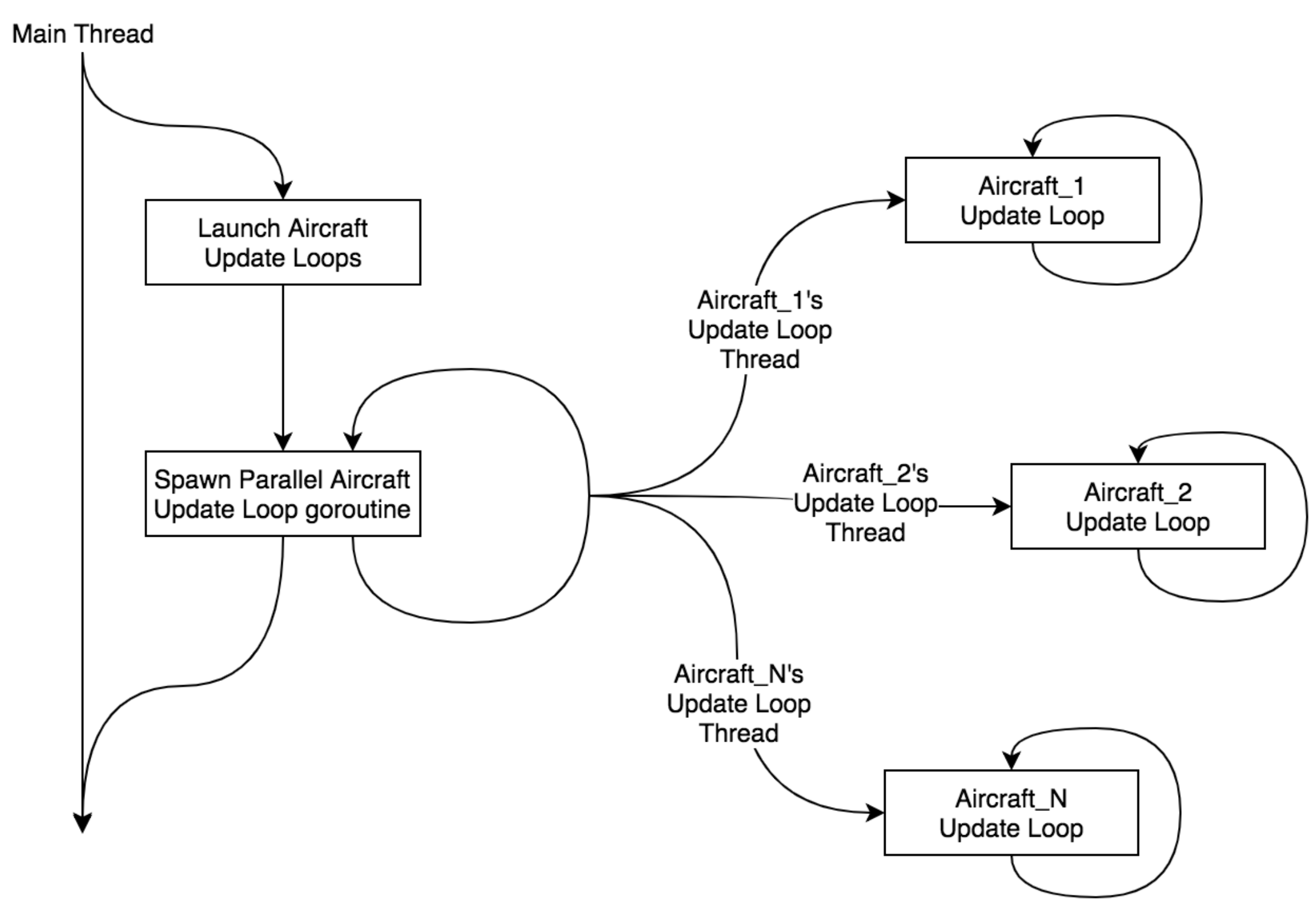


Utilize the UNIX philosophy, "Write programs that do one thing and do it well, write programs that work together."

Dynamics

Processing Nodes
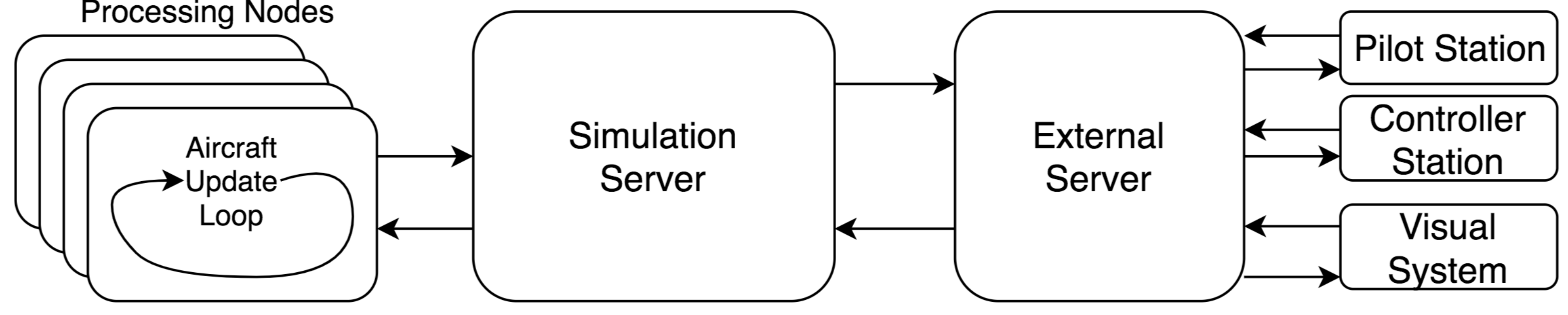

Computes Aircraft

States 
Utilize the UNIX philosophy, "Write programs that do one thing and do it well, write programs that work together."

Dynamics

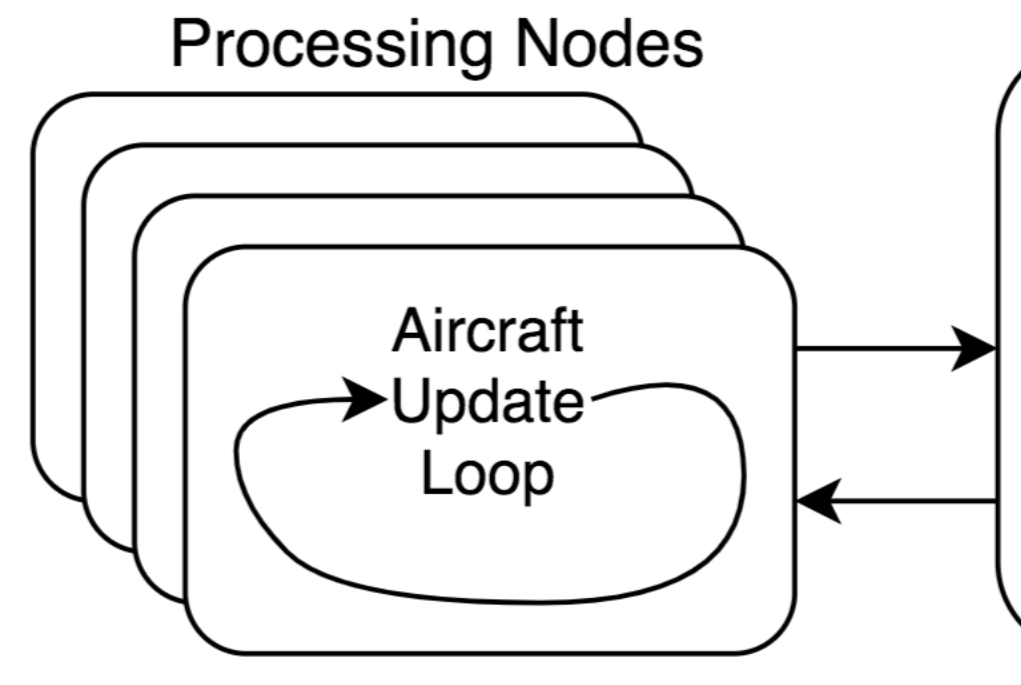

Computes Aircraft States

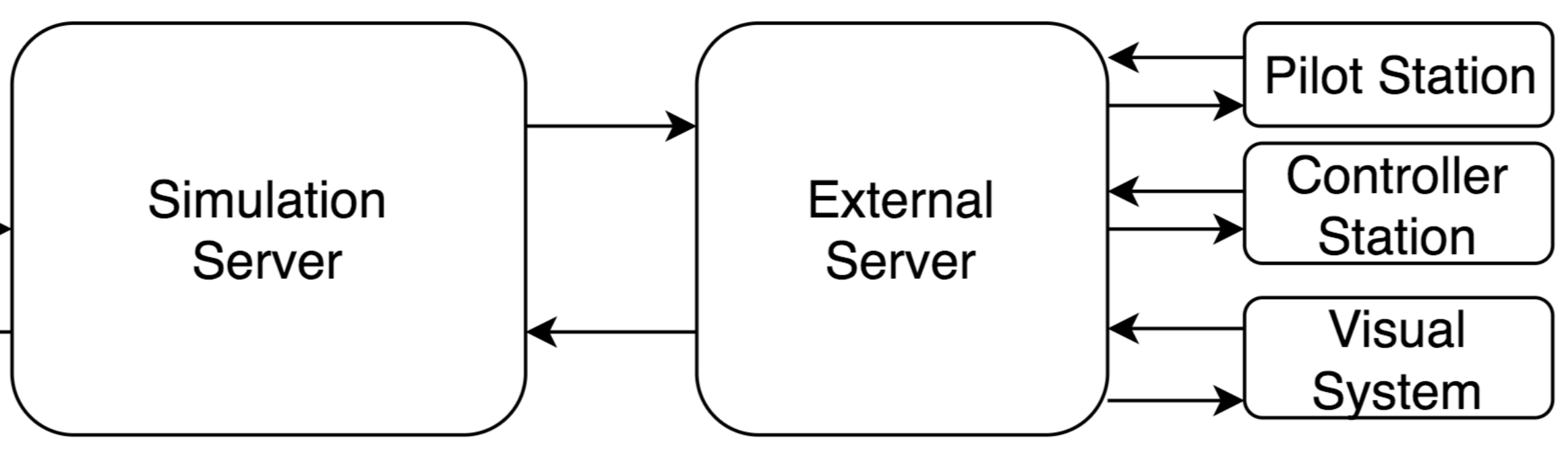

Broker of Messages Simulation Manager
Manages Client Connections Provides Interface to Simulation 
- Clock

- Aircraft Update Loop

- Message Router

- State Update Output
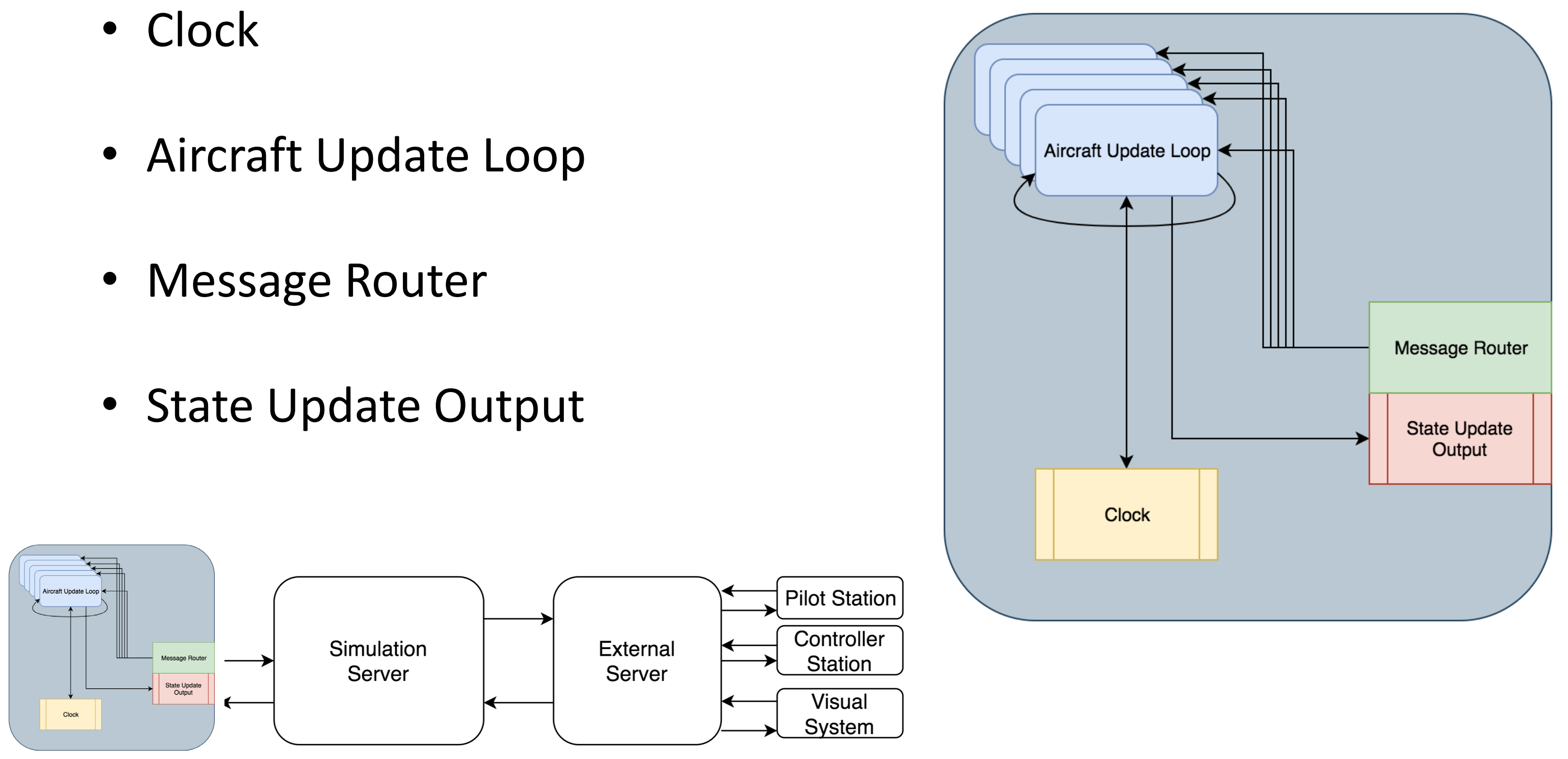
- Message Router

- Aircraft States

- Simulation User Interface
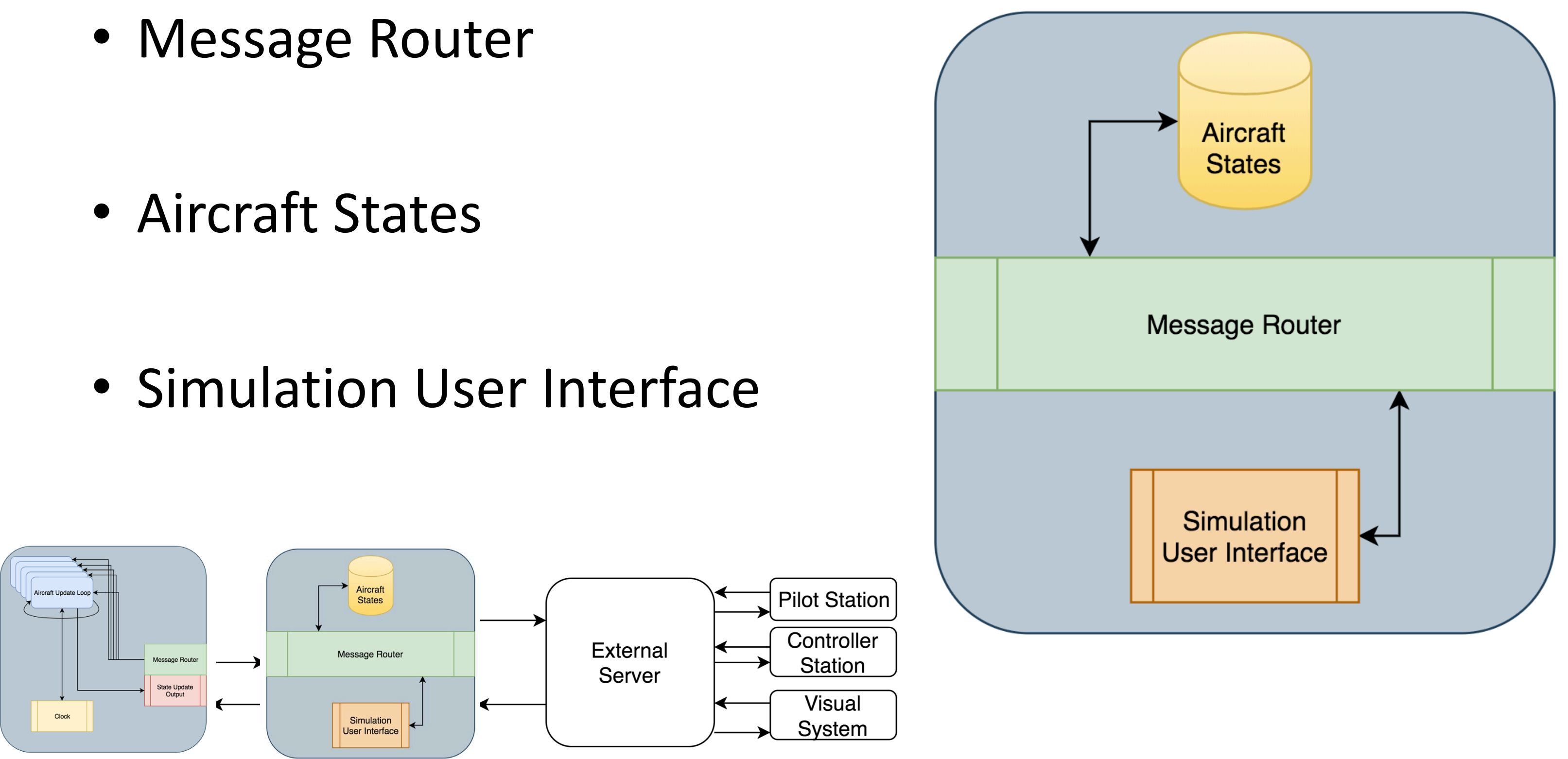
- Simulation Server Router

- Rate Limiter

- Web Server

- General Interface
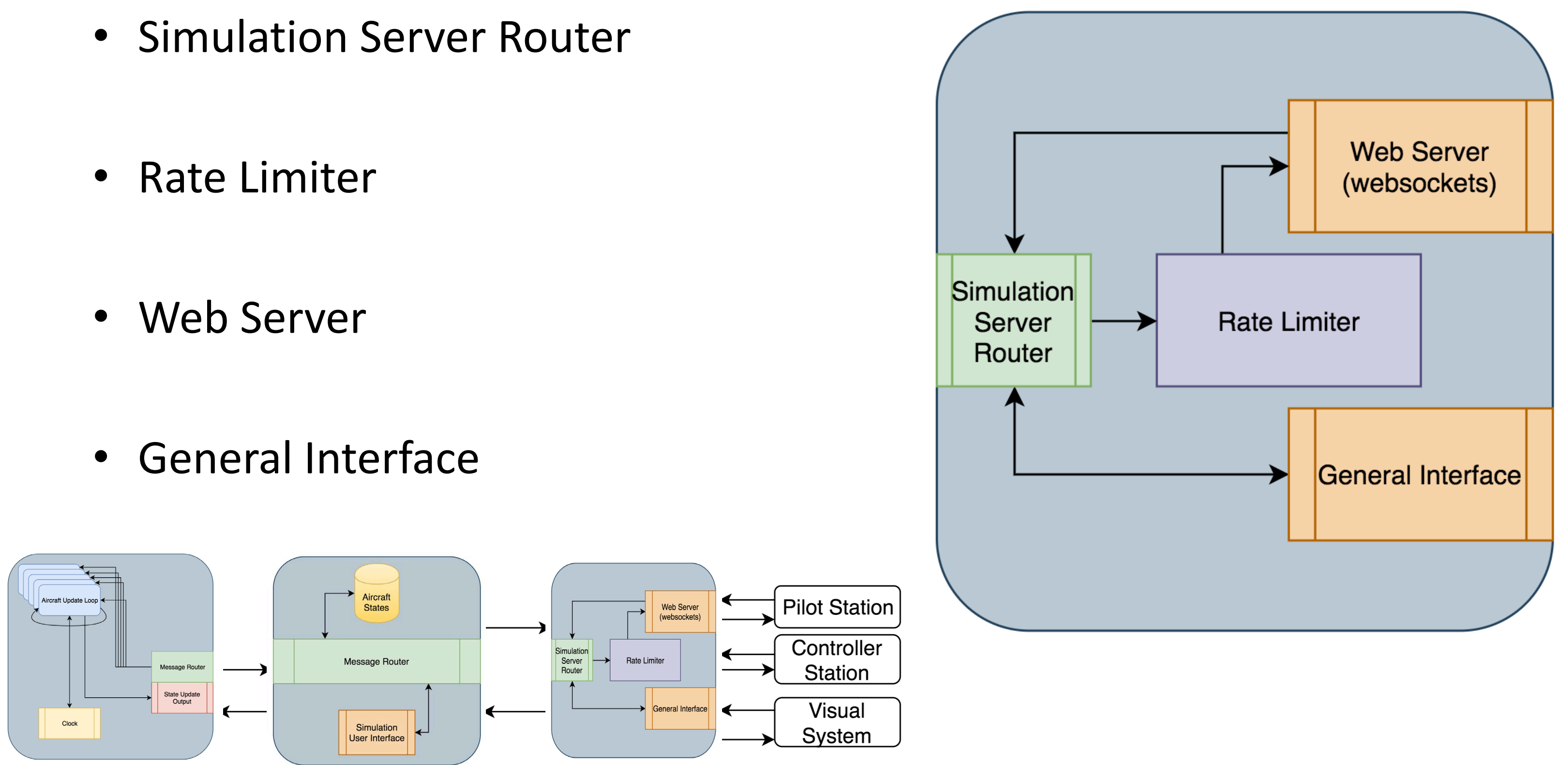


\begin{tabular}{|l|l|l|l|} 
& $\begin{array}{l}\text { Internal Simulation } \\
\text { Communication }\end{array}$ & $\begin{array}{l}\text { Low Bandwidth } \\
\text { Clients }\end{array}$ & $\begin{array}{l}\text { High Bandwidth } \\
\text { Clients }\end{array}$ \\
\hline $\begin{array}{l}\text { Local } \\
\text { Communication }\end{array}$ & UNIX Sockets & $\begin{array}{l}\text { N/A } \\
\text { (Only Distributed) }\end{array}$ & $\begin{array}{l}\text { N/A } \\
\text { (Only Distributed) }\end{array}$ \\
\hline $\begin{array}{l}\text { Distributed } \\
\text { Communication }\end{array}$ & TCP & HTTP/Websockets & TCP \\
\hline
\end{tabular}




\section{NAsA Why WebSockets and HTTP for Pilot and Controller Stations?}

- Browser Web Applications allow for easeof-access from any on-site location or VPN'd client

- WebSockets provide streaming data to support a real-time connection to simulation

- HTTP enables upgrading to websockets, as well as a strong interface for requestresponse communication
Controller

Station

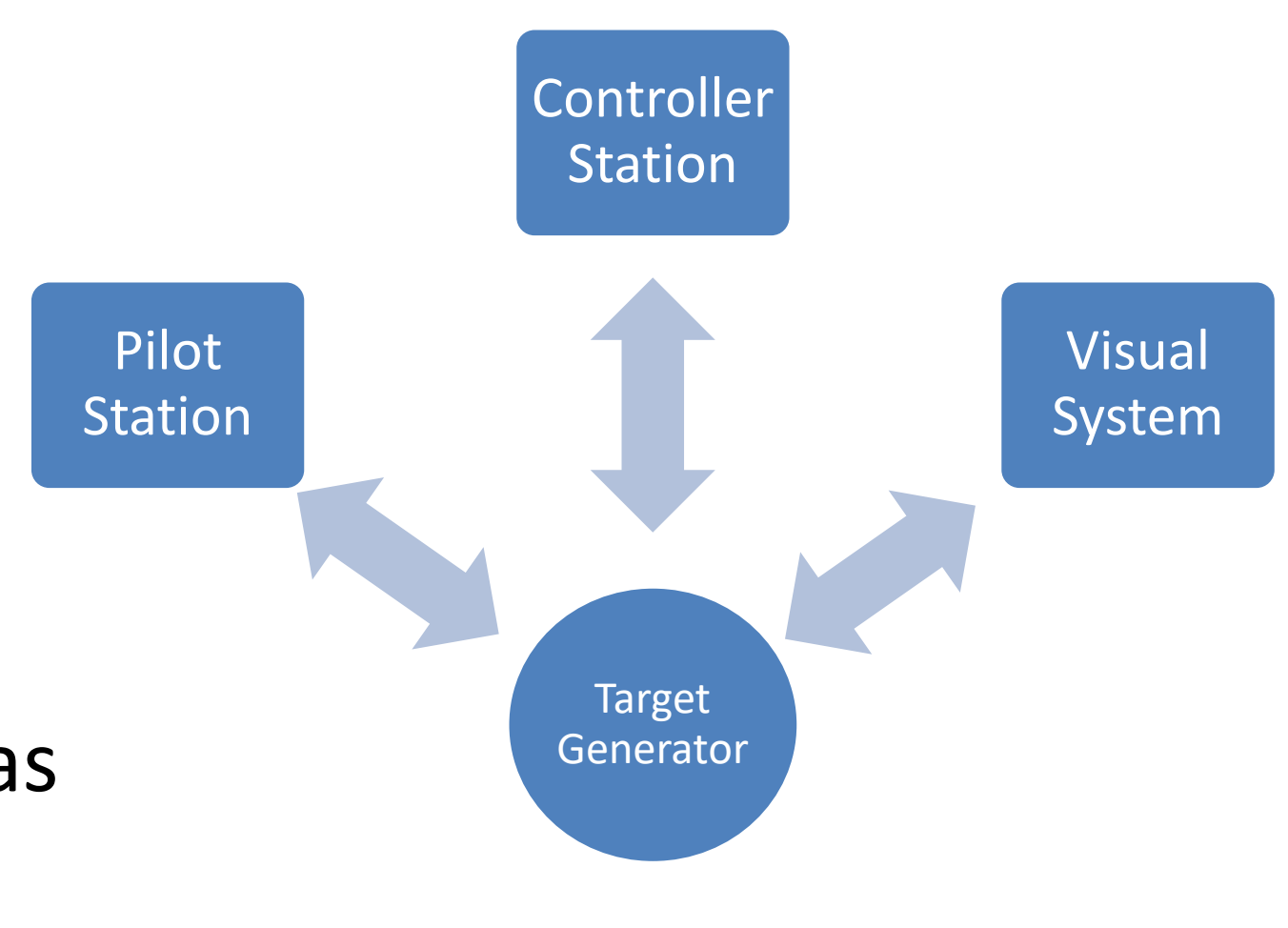


- Current day target generators offer $1-4 \mathrm{~Hz}$, up to 200 Active Aircraft

Prototype Tests:

- Two system configurations

- one all-local simulation

- one distributed system

\begin{tabular}{l|c|c} 
& 400 Aircraft & 1000 Aircraft \\
\hline One Node & $125.4 \mathrm{~Hz}$ & $48.9 \mathrm{~Hz}$ \\
\hline Two Node & $79.7 \mathrm{~Hz}$ & $32.0 \mathrm{~Hz}$
\end{tabular}

- Two loads of traffic

- one 400 aircraft

- one 1000 aircraft 
- One Node Configurations outperformed Two Node Configurations:

- Need to optimize messaging system for TCP

- Develop Clients

- Pilot Station

- Controller Station

- Interface with Visual Systems 
- Prototype demonstrates update rates of $30 \mathrm{~Hz}+$ outperforming current day target generators

- Provides smooth out-the-window visuals

- Supports higher aircraft density

- Expands flexibility for client software

- Improves capabilities for future simulations research 